\title{
Evidence for charm-bottom tetraquarks and the mass dependence of heavy-light tetraquark states from lattice QCD
}

\author{
Anthony Francis, ${ }^{1, *}$ Renwick J. Hudspith, ${ }^{2, \dagger}$ Randy Lewis, ${ }^{2, \ddagger}$ and Kim Maltman ${ }^{2, \S}$ \\ ${ }^{1}$ Theoretical Physics Department, CERN, CH-1211 Geneva 23, Switzerland \\ ${ }^{2}$ Department of Physics and Astronomy, York University, Toronto, Ontario M3J 1P3, Canada
}

(Received 31 October 2018; published 21 March 2019)

\begin{abstract}
We continue our study of heavy-light four-quark states and find evidence from lattice QCD for the existence of a strong-interaction-stable $I\left(J^{P}\right)=0\left(1^{+}\right) u d \bar{c} \bar{b}$ tetraquark with mass in the range of 15 to $61 \mathrm{MeV}$ below $\bar{D} B^{*}$ threshold. Since this range includes the electromagnetic $\bar{D} B \gamma$ decay threshold, current uncertainties do not allow us to determine whether such a state would decay electromagnetically or only weakly. We also perform a study at fixed pion mass, with non-relativistic QCD (NRQCD) for the heavy quarks, simulating $q q^{\prime} \bar{b}^{\prime} \bar{b}$ and $q q^{\prime} \bar{b}^{\prime} \bar{b}^{\prime}$ tetraquarks with $q, q^{\prime}=u d$ or $\ell s$ and variable, unphysical $m_{b^{\prime}}$ in order to investigate the heavy mass dependence of such tetraquark states. We find that the dependence of the binding energy follows a phenomenologically expected form and that, though NRQCD breaks down before $m_{b^{\prime}}=m_{c}$ is reached, the results at higher $m_{b^{\prime}}$ clearly identify the $u d \bar{b}^{\prime} \bar{b}$ channel as the most likely to support a strong-interaction-stable tetraquark state at $m_{b^{\prime}}=m_{c}$. This observation serves to motivate the direct $u d \bar{c} \bar{b}$ simulation. Throughout we use dynamical $n_{f}=2+1$ ensembles with pion masses $m_{\pi}=415$, 299 , and $164 \mathrm{MeV}$ reaching down almost to the physical point, a relativistic heavy quark prescription for the charm quark and NRQCD for the bottom quark(s).
\end{abstract}

DOI: 10.1103/PhysRevD.99.054505

\section{INTRODUCTION}

Many theoretical efforts since the formulation of QCD have hypothesized the existence of exotic states containing four or more quarks and/or antiquarks (for a recent review see [1] and references therein). It is only in the past decade that unambiguously exotic states, including the hiddencharm pentaquark states recently discovered at $\mathrm{LHCb}$ [2] and at least some of the $\mathrm{X}, \mathrm{Y}$ and $\mathrm{Z}$ states [1], which fail to fit into the standard quark model picture, have begun to be observed experimentally. These experimental results have shown that complicated many-quark structures do exist in nature, and the goal for theorists is to investigate why some such multiquark structures are preferred and to elucidate the mechanisms underlying their existence. The mechanisms behind the binding of such configurations should help to provide greater insight into the complex phenomena of QCD in the nonperturbative realm.

\footnotetext{
*anthony.francis@cern.ch

†renwick.james.hudspith@googlemail.com

randy.lewis@yorku.ca

§maltman@yorku.ca
}

Published by the American Physical Society under the terms of the Creative Commons Attribution 4.0 International license. Further distribution of this work must maintain attribution to the author(s) and the published article's title, journal citation, and DOI. Funded by SCOAP.
In this work we use lattice QCD to investigate configurations of two light quarks and two heavy antiquarks in channels expected to be favorable to the formation of bound, exotic tetraquark states. In general, such four-quark bound states have not yet been definitely proven to exist experimentally, but there are indications, both from models and from lattice QCD [3-29], that they should exist. A benefit of employing the lattice approach is that unphysical quark masses can be used as input simulation parameters, allowing for an extended investigation of the underlying binding mechanisms.

In a prior work [29] we predicted the existence of $u d \bar{b} \bar{b}$ and $\ell s \bar{b} \bar{b}$ tetraquarks with quantum numbers $I\left(J^{P}\right)=$ $0\left(1^{+}\right)$and $\frac{1}{2}\left(1^{+}\right)$, respectively, using lattice QCD. The focus on these channels was motivated by features of the splittings in the heavy baryon spectrum. The key observation is that a pair of heavy antiquarks in a color $3_{c}$ configuration will serve as the source of a nearly static color $3_{c}$ field, analogous to that produced by the heavy quark in a singly heavy baryon. From the pattern of splittings in the heavy baryon sector, it is clear that a strong spin-dependent attraction exists for light quark $u d$ or $\ell s$ pairs in Jaffe's [30] "good-diquark" configuration (color $\overline{3}_{c}$ and $I=J=0$ or $I=1 / 2, J=0$, for $u d$ or $\ell s$, respectively). The strength of this attraction, moreover, increases the lighter the light quark mass. In a doubly heavy $I\left(J^{P}\right)=0\left(1^{+}\right)$or $\frac{1}{2}\left(1^{+}\right)$ $q q^{\prime} \bar{Q} \bar{Q}^{\prime}$ tetraquark channel, this good-diquark attraction is 
available to a localized four-quark state, but not to the lowest-lying asymptotic two-meson state in the same channel, where the spin-dependent interactions of the light quarks with their heavy antiquark partners from the same meson are suppressed by the heavy quark mass. The attractive $\bar{Q} \bar{Q}^{\prime} 3_{c}$ color-Coulomb interaction provides a further contribution to binding in the localized tetraquark configuration not available to two separated mesons. This picture leads to the expectation that bound $u d \bar{b} \bar{b}$ and $\ell s \bar{b} \bar{b}$ tetraquark states should exist in the $I\left(J^{P}\right)=0\left(1^{+}\right)$and $\frac{1}{2}\left(1^{+}\right)$channels, with binding of order $150-200 \mathrm{MeV}$ for the former and a reduced binding for the latter. These semiquantitative expectations were confirmed by explicit lattice simulations, in which we found states bound by $\left|\Delta E_{u d \bar{b} \bar{b}}\right|=189(10)(3)$ and $\left|\Delta E_{\ell s \bar{b} \bar{b}}\right|=98(7)(3) \mathrm{MeV}$ relative to the corresponding two-meson thresholds, $B B^{*}$ and $B_{s} B^{*}$, respectively, at the physical mass point. With such binding energies, these states are not only stronginteraction stable, but can, in fact, decay only weakly. Other lattice studies using static prescriptions of the bottom quarks and heavier than physical sea quarks [31-40] analyzing similar quantities have observed attractive potentials in this channel and some [28,41-43] have indicated binding as well.

Since the discovery of the doubly charmed $\Xi_{c c}$ baryon at $\mathrm{LHCb}$ [44], sum rule calculations and phenomenological models (see e.g., [24,25,45,46]) have also led to the identification of these channels as favorable to doubly heavy tetraquark binding. In the model calculations, an important role is also played by the attractive natures of the light-quark spin-dependent interactions and short distance color-Coulomb potential for heavy antiquark pairs in a $3{ }_{c}$ color configuration [1].

Assuming the above picture correctly captures the basic physics involved in the binding observed in the $u d \bar{b} \bar{b}$ and $\ell s \bar{b} \bar{b}$ systems, one should see binding which grows as the heavy quark mass is increased since the short-distance, $3_{c}$ color-Coulomb attraction should scale as the reduced mass of the heavy anti-diquark system. One should also see contributions to the binding which are, to a first approximation, independent of the heavy quark mass corresponding to the good-light-diquark attraction in the static heavy quark limit. Corrections to this limit should produce binding corrections proportional to the inverse of the heavy quark mass. The increase with decreasing heavy-quark mass of the net residual light-heavy spin-dependent attraction in the two-meson (vector-pseudoscalar) threshold will also reduce the tetraquark binding relative to this threshold and produce binding corrections proportional to the inverse of the heavy quark mass.

These qualitative expectations can be tested by extending our previous study to include unphysical values of the masses of one or both of the two heavy antiquarks. The only limitation is the non-relativistic QCD (NRQCD) approach to treating the $\bar{b}$, which breaks down before the charm quark mass is reached. As we will see, the results of this variable-heavy-mass study confirm the picture outlined above. Once this has been established, even though the NRQCD-based approach does not allow us to push this study down to the charm mass, the pattern of bindings obtained as the variable heavy mass (or masses) is (are) decreased below $m_{b}$ can, nonetheless, be used to identify which channel (or channels) involving one or two charmed antiquarks is (are) most likely to support bound tetraquark states. Such information is useful for optimally allocating available computing resources. The results of this study lead us to focus our attention, and direct simulation efforts, on the most favorable of these channels, which turns out to be the $u d \bar{c} \bar{b}$ channel.

In this paper we will first detail the variable heavy mass study outlined above, and then we discuss the results of our direct simulations of the $u d \bar{c} \bar{b}$ channel. Throughout, we will use the same three dynamical, fixed lattice spacing, $n_{f}=2+1$ PACS-CS ensembles employed in our previous $u d \bar{b} \bar{b}$ study. These ensembles have pion masses $m_{\pi}=164$, 299 , and $415 \mathrm{MeV}$. A relativistic prescription will be used for the charm quark and, as before, NRQCD for the bottom quark. Evidence is presented for the existence of an $I\left(J^{P}\right)=0\left(1^{+}\right) u d \bar{c} \bar{b}$ tetraquark bound with respect to the lowest noninteracting two-meson threshold, $\bar{D} B^{*}$, in this channel.

For the variable-heavy-mass study, we will focus our attention on the ensemble with $m_{\pi}=299 \mathrm{MeV}$ and study the heavy anti-diquark mass dependence for unphysical tetraquark candidates $q q^{\prime} \bar{Q} \bar{Q}^{\prime}$, with $q=u, q^{\prime}=d$, s, and either $\bar{Q}^{\prime} \neq \bar{Q}$ or $\bar{Q}^{\prime}=\bar{Q}$.

\section{PHENOMENOLOGY OF HEAVY-LIGHT TETRAQUARKS}

We focus on heavy-light tetraquark candidates $q q^{\prime} \bar{Q} \bar{Q}^{\prime}$ that can be pictured as a combination of a "good" lightdiquark $q q^{\prime}$ and heavy anti-diquark $\bar{Q} \bar{Q}^{\prime}$ with $q q^{\prime}=u d$, or $\ell s$ and $Q, Q^{\prime}=b$ or $c$. The color $\overline{3}_{c}, J=0$, flavorantisymmetric good-light-diquark configuration is accessible only when the heavy anti-diquark is in color $3_{c}$. Assuming no spatial excitation between the heavy antiquarks, the heavy anti-diquark spin is necessarily $J_{h}=1$ when $Q=Q^{\prime}$. The favored tetraquark configuration is then $J^{P}=1^{+}$.

In the limit of infinitely heavy $Q, Q^{\prime}, m_{Q, Q^{\prime}} \rightarrow \infty$, the attractive nature of the color-Coulomb potential guarantees a bound ground state of the $q q^{\prime} \bar{Q} \bar{Q}^{\prime}$ type [47-49]. Whether a bound state is realized away from this limit, in particular, when $Q, Q^{\prime}$ are charm or bottom quarks, depends on the details of nonperturbative effects in such systems.

The phenomenological arguments outlined in the previous section, based on observed splitting patterns in the heavy baryon system, were shown in Ref. [29] to suggest the likelihood of the existence of tetraquark bound states of 
the $q q^{\prime} \bar{b} \bar{b}$ type with tetraquark binding increasing with decreasing light quark mass(es).

The lattice results of Ref. [29] not only confirmed the existence of these bound states, but produced binding energies for physical light quark masses in line with those expected based on the heavy baryon spectrum arguments. Assuming the picture underlying this successful prediction is correct, and the good-diquark contribution to binding indeed increases with decreasing light quark mass, this implies it is imperative to have access to light quark masses as close to physical as possible in lattice simulations of such tetraquark channels. This is a firm prediction of this binding mechanism, one that may seem counterintuitive given the common experience with lattice calculations in other channels, for example, in the study of multibaryon states, where a decrease in constituent quark masses also decreases the binding energy (for a collection of recent results and presentation of the issues faced see [50] and references therein).

It is possible to further test the qualitative physical picture underlying this understanding of the $u d \bar{b} \bar{b}$ and $\ell s \bar{b} \bar{b}$ tetraquark binding observed in Ref. [29] by studying related systems with variable heavy antiquark mass(es). This study is carried out using the same NRQCD action used previously, in Ref. [51], for the physical bottom quark case. A brief outline of the NRQCD framework, together with details of the implementation of the variable $b^{\prime}$ mass, are provided in Appendix A. The NRQCD heavy-mass parameter, $m_{Q}$, was tuned by measuring the dispersion relation of the spin-averaged $\Upsilon$ and $\eta_{b}$. We also computed static propagators, allowing the extrapolation of $m_{b^{\prime}}$ to $\infty$ to be carried out for the $Q^{\prime} \neq Q$ case. For the variable heavy mass study, we focus, to be specific, on the intermediate ensemble, $E_{M}$, with $m_{\pi}=299 \mathrm{MeV}$ and $m_{\pi} L=4.4$, and consider unphysical bottom quark masses $\simeq 6.29$, 4.40, $1.93,1.46,0.85,0.68,0.64$, and 0.60 times the physical bottom quark mass. Lower values are not accessible in this approach due to the breakdown of the NRQCD approximation. Denoting such unphysical bottom quarks by $b^{\prime}$, we investigate $q q^{\prime} \bar{b} \bar{b}^{\prime}$ and $q q^{\prime} \bar{b}^{\prime} \bar{b}^{\prime}$ tetraquark channels.

Given the qualitative physical picture outlined above, we expect there to be a contribution to tetraquark binding from the color-Coulomb attraction between the two heavy antiquarks in the color $3_{c}$ configuration which scales linearly with the reduced mass of the heavy anti-diquark system. There should also be a contribution to the binding which, for a given light-diquark channel, should be independent of the heavy quark masses, reflecting the attractive nature of the good-light-diquark configuration in the infinite heavy quark mass limit. Finally, there should be contributions to the binding resulting from the presence of residual heavy-light interactions, scaling as the inverse of the heavy quark mass(es), in both the tetraquark and twomeson threshold states. Contributions of the former type should scale as $\frac{1}{m_{h 1}}+\frac{1}{m_{h 2}}$ for tetraquark states with heavy antiquark masses $m_{h 1}$ and $m_{h 2}$. Residual interactions between a heavy quark and light diquark are also present in the heavy baryon systems. Comparing the $\Sigma_{h}-\Lambda_{h}$ and $\Xi_{h}^{\prime}-\Xi_{h}$ splittings for $h=b, c$, one finds heavy baryon residual interactions depending on both the inverse of the heavy quark mass and the type ( $u d$ or $\ell s$ ) of light diquark. We thus expect the coefficient of $\frac{1}{m_{h 1}}+\frac{1}{m_{h 2}}$ for the residual heavy-light tetraquark interactions to be different for tetraquarks containing $u d$ and $\ell s$ good diquarks. With the ratio of observed charm and bottom vector-pseudoscalar splittings in good agreement with expectations based on the assumption that these scale as the inverse of the relevant heavy quark mass, the contributions to tetraquark binding from residual heavy-light interactions in the corresponding two-meson threshold state can be directly determined from the observed $B^{*}-B, B_{s}^{*}-B_{s}, D^{*}-D$, and $D_{s}^{*}-D_{s}$ splittings, bearing in mind that the correct two-meson threshold must be chosen. Thus, for example, denoting by $V^{\prime}$ and $P^{\prime}$ the $\bar{b}^{\prime} \ell$ vector and pseudoscalar states, one has that, for tetraquarks of the $u d \bar{b} \bar{b}^{\prime}$ type, the relevant threshold is $B^{*} P^{\prime}$ for $m_{b^{\prime}}<m_{b}$ but $B V^{\prime}$ for $m_{b^{\prime}}>m_{b}$. We assume that the observed $1 / m_{h}$ scaling of the bottom and charm vector-pseudoscalar splittings persists for the variable- $b$ mass $V^{\prime}-P^{\prime}$ splittings. The two-meson threshold contributions for a given physical-to-variable $b$ quark mass ratio, $r=m_{b} / m_{b^{\prime}}$, are then fixed by the observed charm/bottom meson splittings and depend on $m_{h 1}$ and $m_{h 2}$ in a manner that varies depending on the relation between these two masses. We use that the vector meson, $V^{\prime}$, and pseudoscalar meson, $P^{\prime}$, lie, respectively, $\frac{1}{4}\left(m_{V^{\prime}}-m_{P^{\prime}}\right)$ above and $\frac{3}{4}\left(m_{V^{\prime}}-m_{P^{\prime}}\right)$ below the spin average of the $V^{\prime}$ and $P^{\prime}$ masses.

Taking these expectations into account, and writing the results in terms of the physical-to-variable mass ratio $r=m_{b} / m_{b^{\prime}}$, one expects to obtain a good-quality fit to the binding energies of tetraquarks with at least one unphysical variable-mass antiquark using an expression having the form

$$
\Delta E=\frac{C_{0}}{2 r}+C_{1}^{u d}+C_{2}^{u d}(2 r)+23 \mathrm{MeV} r
$$

for the $u d \bar{b}^{\prime} \bar{b}^{\prime}$ case, where the first term represents the Coulomb binding contribution, the second the good- $u d$ diquark attraction, the third the residual heavy-light interactions in the tetraquark state, and the fourth the two-meson threshold contribution. The numerical value appearing in the fourth term follows from the observed meson splittings. Similarly, for the $u d \bar{b}^{\prime} \bar{b}$ case, one expects the form

$$
\Delta E=\frac{C_{0}}{1+r}+C_{1}^{u d}+C_{2}^{u d}(1+r)+(34 \mathrm{MeV}-11 \mathrm{MeV} r)
$$


to provide a good representation for $m_{b^{\prime}}>m_{b}$ and the form

$$
\Delta E=\frac{C_{0}}{1+r}+C_{1}^{u d}+C_{2}^{u d}(1+r)+(34 \mathrm{MeV} r-11 \mathrm{MeV})
$$

to provide a good representation for $m_{b^{\prime}}<m_{b}$. The corresponding expectations for the cases involving an $\ell s$, rather than $u d$, good diquark are

$$
\Delta E=\frac{C_{0}}{2 r}+C_{1}^{\ell s}+C_{2}^{\ell s}(2 r)+24 \mathrm{MeV} r
$$

for $\ell s \bar{b}^{\prime} \bar{b}^{\prime}$

$\Delta E=\frac{C_{0}}{1+r}+C_{1}^{\ell s}+C_{2}^{\ell s}(1+r)+(34 \mathrm{MeV}-12 \mathrm{MeV} r)$

for $\ell s \bar{b}^{\prime} \bar{b}$ with $m_{b^{\prime}}>m_{b}$, and

$\Delta E=\frac{C_{0}}{1+r}+C_{1}^{\ell s}+C_{2}^{\ell s}(1+r)+(36 \mathrm{MeV} r-11 \mathrm{MeV})$

for $\ell s \bar{b}^{\prime} \bar{b}$ with $m_{b^{\prime}}<m_{b}$.

For the variable- $b$-mass study just described, details of the setup and determination of the resulting tetraquark binding energies may be found in Appendixes A and B. Figure 1 and Table I display these results for $m_{b^{\prime}}$ running from 6.29 to 0.60 times $m_{b}$. The restriction to $m_{b^{\prime}} \geq$ $0.60 m_{b}$ is designed to ensure that the $b^{\prime}$ masses considered are all sufficiently heavy that the NRQCD approximation is

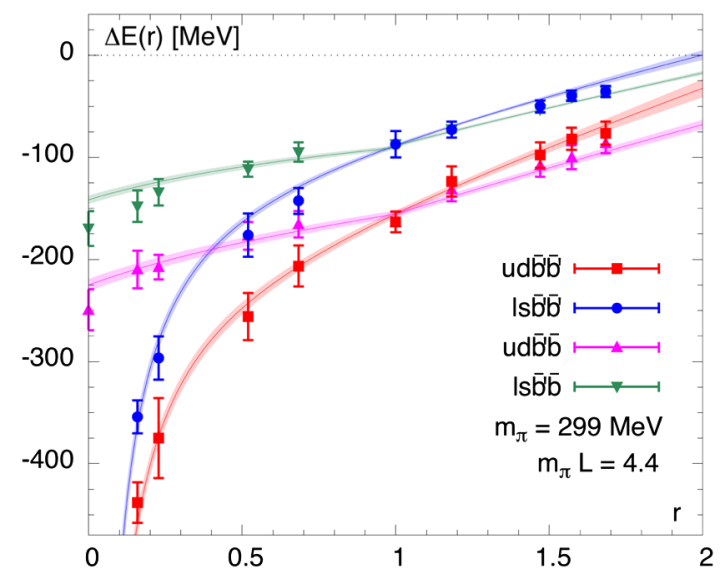

FIG. 1. The dependence on the heavy-quark mass ratio, $r=m_{\text {bare }}^{b} / m_{\text {bare }}^{b^{\prime}}$, of the binding energies for the $u d \bar{b}^{\prime} \bar{b}, u d \bar{b}^{\prime} \bar{b}^{\prime}$, $\ell s b^{\prime} b$, and $\ell s b^{\prime} b^{\prime}$ channels. The results for each channel are separately fit to the phenomenologically motivated $\Delta E\left(m_{Q}\right)$ ansatze detailed in Eqs. (1)-(6) of the text.
TABLE I. Table of binding energies determined in the nonrelativistic regime. Note the values denoted with "**" were calculated previously in [51].

\begin{tabular}{lcccc}
\hline \hline & \multicolumn{4}{c}{$\Delta E[\mathrm{MeV}]$} \\
\cline { 2 - 5 }$m_{b^{\prime}} / m_{b}$ & $u d \bar{b}^{\prime} \bar{b}^{\prime}$ & $u d \bar{b}^{\prime} \bar{b}$ & $\ell s \bar{b}^{\prime} \bar{b}^{\prime}$ & $\ell s \bar{b}^{\prime} \bar{b}$ \\
\hline 0.594 & $-76(11)$ & $-86(10)$ & $-35(5)$ & $-35(4)$ \\
0.636 & $-82(11)$ & $-100(12)$ & $-39(5)$ & $-41(3)$ \\
0.680 & $-98(13)$ & $-108(11)$ & $-50(6)$ & $-53(2)$ \\
0.846 & $-123(15)$ & $-131(12)$ & $-73(8)$ & $-72(3)$ \\
1.000 & $-163(8)^{*}$ & $\ldots$ & $-94(9)^{*}$ & $\ldots$ \\
1.463 & $-206(20)$ & $-166(13)$ & $-143(13)$ & $-95(10)$ \\
1.928 & $-256(23)$ & $-177(14)$ & $-176(21)$ & $-112(7)$ \\
4.935 & $-375(39)$ & $-208(12)$ & $-296(21)$ & $-134(13)$ \\
6.287 & $-438(20)$ & $-210(18)$ & $-354(16)$ & $-148(15)$ \\
$\infty$ & $\ldots$ & $-249(30)$ & $\ldots$ & $-170(17)$ \\
\hline \hline
\end{tabular}

reliable. The same $2 \times 2$ generalized eigenvalue problems (GEVPs) used in Ref. [51] are employed for the $u d \bar{b}^{\prime} \bar{b}^{\prime}$ and $\ell s \bar{b}^{\prime} \bar{b}^{\prime}$ channels, while new $3 \times 3$ GEVPs, described in more detail in the next section, are used for $u d \bar{b}^{\prime} \bar{b}$ and $\ell s \bar{b}^{\prime} \bar{b}$. The results of a fit to these data using the forms detailed above are shown in Fig. 1 and Table II. The success of this fit in describing tetraquark binding energies over a wide range of variable heavy quark masses confirms that the physical picture underlying those fit forms successfully captures the main features responsible for the binding observed in these systems.

While the use of NRQCD precludes extending the results of the variable- $b$-mass study down to the charm mass, the pattern of binding energies shown in Fig. 1 clearly points to the $u d \bar{c} \bar{b}$ channel as by far the most likely among the four channels, $u d \bar{c} \bar{b}, \ell s \bar{c} \bar{b}, u d \bar{c} \bar{c}$, or $\ell s \bar{c} \bar{c}$, in which one or both of the $\bar{b}$ antiquarks in $u d \bar{b} \bar{b}$ is replaced by $\bar{c}$, to support a strong-interaction-stable bound state. A naive extrapolation of the results for this channel, moreover, produces a result very near the $\bar{D} B^{*}$ threshold, strongly motivating direct $u d \bar{c} \bar{b}$ simulations using a relativistic action for the charm quark. Similar naive extrapolations of the variable-heavy-mass results suggest none of the other three channels is likely to support a strong-interactionstable bound state. The results of the variable- $b$-mass study thus allow us to focus in what follows on the $u d \bar{c} \bar{b}$ channel, leaving detailed simulations of the other channels for a subsequent work.

TABLE II. Fit results for the constants parametrizing the heavyquark mass dependence of tetraquark binding energies on the ensemble $E_{M}$ with pion mass $m_{\pi}=299 \mathrm{MeV}$.

\begin{tabular}{lcccc}
\hline \hline$C_{0}$ & $C_{1}^{\text {ud }}$ & $C_{2}^{\text {ud }}$ & $C_{1}^{\ell s}$ & $C_{2}^{\ell^{s}}$ \\
\hline$-82(6)$ & $-217(14)$ & $40(5)$ & $-116(10)$ & $22(3)$ \\
\hline \hline
\end{tabular}




\section{LATTICE CORRELATORS AND OPERATORS}

The generic form of a lattice QCD correlation function for a particle at rest, i.e., $\mathbf{p}=0$, in Euclidean time is given by

$$
\begin{aligned}
C_{\mathcal{O}_{1} \mathcal{O}_{2}}(t) & =\sum_{\mathbf{x}}\left\langle\mathcal{O}_{1}(\mathbf{x}, t) \mathcal{O}_{2}(\mathbf{0}, 0)^{\dagger}\right\rangle \\
& =\sum_{n} \frac{\left\langle 0\left|\mathcal{O}_{1}\right| n\right\rangle\left\langle n\left|\mathcal{O}_{2}\right| 0\right\rangle}{2 E_{n}} e^{-E_{n} t},
\end{aligned}
$$

with the interpolating operators $\mathcal{O}_{i}$ being chosen to have the quantum numbers of the continuum state to be studied. For example, the simplest local meson operator is

$$
\mathcal{O}_{m}(x)=\bar{q}_{a}^{\alpha}(x) \Gamma^{\alpha \beta} q_{a}^{\prime \beta}(x),
$$

where upper (Greek) indices denote Dirac spin and lower (Roman) color. The $q$ and $q^{\prime}$ represent the constituent quark flavors.

In the case of the $\overline{3}_{F}, J^{P}=1^{+}$tetraquarks, the relevant noninteracting two-meson thresholds are given by the sums of the lowest-lying pseudoscalar (P) $\Gamma=\gamma_{5}$ and vector $(\mathrm{V})$ $\Gamma=\gamma_{i}$ meson masses. Table III provides a list of these thresholds for the cases of interest here.

Given the phenomenological picture of the previous sections, a natural first choice of the interpolating operator for $q q^{\prime} \bar{Q} \bar{Q}^{\prime}$-type tetraquarks is one with a diquark-anti-diquark structure. Using the epsilon identity $\epsilon_{a b c} \epsilon_{d e c}=\delta_{a d} \delta_{b e}-$ $\delta_{a e} \delta_{b d}$, the local version of this operator takes the form

$$
\begin{aligned}
D(x)= & \left(\left(q_{a}^{\alpha}(x)\right)^{T}\left(C \gamma_{5}\right)^{\alpha \beta}{q^{\prime}}_{b}^{\prime \beta}(x)\right) \\
& \times\left[\bar{Q}_{a}^{\kappa}(x)\left(C \gamma_{i}\right)^{\kappa \rho}\left(\bar{Q}_{b}^{\prime \rho}(x)\right)^{T}\right. \\
& \left.-\bar{Q}_{b}^{\kappa}(x)\left(C \gamma_{i}\right)^{\kappa \rho}\left(\bar{Q}_{a}^{\prime \rho}(x)\right)^{T}\right],
\end{aligned}
$$

where $C=i \gamma_{y} \gamma_{t}$ is the charge-conjugation matrix. This operator has $\bar{Q} \bar{Q}^{\prime}$ color $3_{c}$, spin 1 , and light-quark flavorspin-color $\left(\overline{3}_{F}, 0, \overline{3}_{c}\right)$.

A second possible local operator is one whose discrete structure is meson-meson-like. For tetraquark channels with $Q=Q^{\prime}$ this could be

$$
\begin{aligned}
M(x)= & \left(\bar{Q}_{a}^{\alpha}(x) \gamma_{5}^{\alpha \beta} q_{a}^{\beta}(x)\right)\left(\bar{Q}_{b}^{\kappa}(x) \gamma_{i}^{\kappa \rho} q_{b}^{\prime \rho}(x)\right) \\
& -\left(\bar{Q}_{a}^{\alpha}(x) \gamma_{5}^{\alpha \beta} q_{a}^{\prime \beta}(x)\right)\left(\bar{Q}_{b}^{\kappa}(x) \gamma_{i}^{\kappa \rho} q_{b}^{\rho}(x)\right) .
\end{aligned}
$$

TABLE III. Lowest two-meson thresholds for each of the flavor antisymmetric, $J^{P}=1^{+}$tetraquark channels.

\begin{tabular}{lccc}
\hline \hline Tetraquark & Threshold & $\mathcal{O}_{m}=P(x)$ & $\mathcal{O}_{m}=V(x)$ \\
\hline$u d \bar{b} \bar{b}$ & $B B^{*}$ & $\bar{b}_{a}^{\alpha}(x) \gamma_{5}^{\alpha \beta} u_{a}^{\beta}(x)$ & $\bar{b}_{a}^{\alpha}(x) \gamma_{i}^{\alpha \beta} d_{a}^{\beta}(x)$ \\
$\ell s \bar{b} \bar{b}$ & $B_{s} B^{*}$ & $\bar{b}_{a}^{\alpha}(x) \gamma_{5}^{\alpha \beta} s_{a}^{\beta}(x)$ & $\bar{b}_{a}^{\alpha}(x) \gamma_{i}^{\alpha \beta} d_{a}^{\beta}(x)$ \\
$u d \bar{c} \bar{b}$ & $\bar{D} B^{*}$ & $\bar{c}_{a}^{\alpha}(x) \gamma_{5}^{\alpha \beta} u_{a}^{\beta}(x)$ & $\bar{b}_{a}^{\alpha}(x) \gamma_{i}^{\alpha \beta} d_{a}^{\beta}(x)$ \\
\hline \hline
\end{tabular}

When $Q \neq Q^{\prime}$, a second local flavor antisymmetric, $J^{P}=1^{+}$meson-meson-like combination can also be constructed. Suppressing the spin indices, the two possible "meson-meson" interpolating operators in this case are

$$
\begin{aligned}
& M_{1}(x)=\left(\bar{Q}_{a} \gamma_{5} q_{a}\right)\left(\bar{Q}_{b}^{\prime} \gamma_{i} q_{b}^{\prime}\right)-\left(\bar{Q}_{a} \gamma_{5} q_{a}^{\prime}\right)\left(\bar{Q}_{b}^{\prime} \gamma_{i} q_{b}\right), \\
& M_{2}(x)=\left(\bar{Q}_{a}^{\prime} \gamma_{5} q_{a}\right)\left(\bar{Q}_{b} \gamma_{i} q_{b}^{\prime}\right)-\left(\bar{Q}_{a}^{\prime}{ }_{a} \gamma_{5} q_{a}^{\prime}\right)\left(\bar{Q}_{b} \gamma_{i} q_{b}\right) .
\end{aligned}
$$

With these interpolating operators, there are several options to study the ground state energies of the proposed tetraquarks channels. One is to form the so-called binding correlator, the ratio of tetraquark correlation functions to the product of correlation functions, $C_{P P}(t)$ and $C_{V V}(t)$, of the pseudoscalar and vector mesons making up the corresponding noninteracting two-meson threshold in the channel in question:

$$
G_{\mathcal{O}_{1} \mathcal{O}_{2}}(t)=\frac{C_{\mathcal{O}_{1} \mathcal{O}_{2}}(t)}{C_{P P}(t) C_{V V}(t)} .
$$

This definition is beneficial in the present study as the additive mass renormalization for NRQCD quarks explicitly cancels.

The binding correlator behaves, for large $t$, as $\sim e^{-\Delta E t}$, with $\Delta E=m_{0}-m_{V}-m_{P}$, where $m_{0}$ is the ground state mass in the channel. Observing a binding correlator increasing with increasing $t$ thus signals the existence of a ground state lighter than the corresponding two-meson threshold and provides compelling evidence for a bound tetraquark state. The exponential nature of this growth, which will become evident for large enough $t$, will appear linear for $t$ significantly smaller than $1 /|\Delta E|$.

In the binding correlator one might also gain a signal through cancellations between the two-meson and tetraquark fluctuations. At the same time, however, forming the binding correlator may introduce a difficult-to-control systematic through contamination of the tetraquark signal with residual excited state effects originating from the twomeson correlators. The potential problem is that ground state saturation might occur at different lattice times for each of the three different correlation functions entering the binding correlator. The binding correlator plateau will then depend on the slowest plateauing of the three constituent correlators.

To handle (and quantify, if present) this effect, we also compute the individual correlation functions and effective energies for the tetraquark candidates and two-meson threshold combination(s). This is especially important, since we expect a significantly smaller binding energy for $u d \bar{c} \bar{b}$ tetraquarks than was observed for the $u d \bar{b} \bar{b}$ channel. An unambiguous determination of the ground state energies is thus essential. To combine the two mesons we compute the product $C_{P P}(t) C_{V V}(t)$. The resulting mass from a single-exponential fit to this combination for the case of the $\bar{D} B^{*}$ threshold is given in Table IV. Note, 
TABLE IV. Overview of our ensemble parameters (see also [29,51,53]). These configurations [54] use the Iwasaki gauge action with $\beta=1.9$ and nonperturbatively tuned clover coefficient $c_{S W}=1.715$. The $\bar{D} B^{*}$ threshold is extracted from a singleexponential fit to the product of the two relevant $\bar{D}$ - and $B^{*}$-meson correlators. Due to NRQCD's additive mass renormalization, these values have not been converted into physical units.

\begin{tabular}{lccc}
\hline \hline & \multicolumn{3}{c}{ Ensemble parameters } \\
\cline { 2 - 4 } Label & $E_{H}$ & $E_{M}$ & $E_{L}$ \\
\hline Extent & $32^{3} \times 64$ & $32^{3} \times 64$ & $32^{3} \times 64$ \\
$a^{-1}[\mathrm{GeV}][52]$ & $2.194(10)$ & $2.194(10)$ & $2.194(10)$ \\
$\kappa_{l}$ & 0.13754 & 0.13770 & 0.13781 \\
$\kappa_{s}$ & 0.13640 & 0.13640 & 0.13640 \\
$a m_{\pi}$ & $0.18928(36)$ & $0.13618(46)$ & $0.07459(54)$ \\
$m_{\pi} L$ & 6.1 & 4.4 & 2.4 \\
$a E_{\bar{D} B^{*}}[\mathrm{GeV}]$ & $1.3588(17)$ & $1.3367(9)$ & $1.3095(12)$ \\
$M_{J / \Psi}[\mathrm{GeV}]$ & $3.0862(2)$ & $3.0847(2)$ & $3.0685(11)$ \\
$M_{\Upsilon}[.528(79)$ & $9.488(71)$ & $9.443(76)$ \\
Configurations & 400 & 800 & 195 \\
Measurements & 4000 & 6400 & 9360 \\
\hline \hline
\end{tabular}

however, that in this case the NRQCD additive mass renormalization does not drop out and the results are given in lattice units with this shift included.

\section{A. Variational analysis}

With access to several operators with the same quantum numbers, a variational analysis can be used to determine the ground and excited state energies. In the case $\bar{Q}=\bar{Q}^{\prime}$, this analysis involves the $2 \times 2$ matrix

$$
F(t)=\left(\begin{array}{ll}
G_{D D}(t) & G_{D M}(t) \\
G_{M D}(t) & G_{M M}(t)
\end{array}\right) .
$$

When $\bar{Q} \neq \bar{Q}^{\prime}$, there are now two meson-meson interpolating operators, allowing the use of the $3 \times 3$ matrix

$$
F(t)=\left(\begin{array}{ccc}
G_{D D}(t) & G_{D M_{1}}(t) & G_{D M_{2}}(t) \\
G_{M_{1} D}(t) & G_{M_{1} M_{1}}(t) & G_{M_{1} M_{2}}(t) \\
G_{M_{2} D}(t) & G_{M_{2} M_{1}}(t) & G_{M_{2} M_{2}}(t)
\end{array}\right) .
$$

Given the matrix $F(t)$, one can solve the GEVP for some reference time $t_{0}{ }^{1}$

$$
F(t) \nu\left(t, t_{0}\right)=\lambda_{i}(t) F\left(t_{0}\right) \nu\left(t, t_{0}\right),
$$

where $\nu$ are the (generalized) eigenvectors and $\lambda_{i}(t)$ the eigenvalues. The solution of the GEVP gives independent eigenvalues corresponding to different states in the system,

\footnotetext{
${ }^{1}$ We monitor $t_{0}$ to make sure our ground state mass evaluation is stable. We find $t_{0} / a=4$ to be a reasonable choice.
}

$$
\lambda_{i}(t)=A_{i} e^{-\Delta E_{i}\left(t-t_{0}\right)} .
$$

In this work the aim will be to determine the ground state of the system $\left(\lambda_{1}\right)$. As such, a $2 \times 2$ or $3 \times 3$ matrix should suffice, so long as the chosen operators have good overlap with the desired ground state.

\section{NUMERICAL SETUP}

Throughout this work the calculations are performed on three $n_{f}=2+1$, clover-improved [55], Iwasaki gauge [56], PACS-CS ensembles introduced in [54] and which we label by $E_{H}, E_{M}$, and $E_{L}$. The lattice spacing is [52] $a^{-1}=2.194(10) \mathrm{GeV}(a=0.090 \mathrm{fm})$ for all three ensembles. We use a partially quenched strange quark tuned to the (connected) $\phi$ meson [57], which gives a near-physical kaon mass in the chiral limit. The labeling (and pion masses) of the ensembles are consistent with those used in our previous work $[29,51,53]$. In the valence sector we use Coulomb gauge-fixed wall sources using the fourier accelerated conjugate gradient (FACG) algorithm [58], as before. We put sources at multiple time positions and compute propagators for light, strange, and charm quarks using a modified deflated schwartz alternating procedure (SAP) solver [59]. An overview of the lattice parameters and ensemble properties can be found in Table IV.

To reliably handle charm quarks on these lattices we employ a relativistic heavy quark (RHQ) action [60-63], in particular, the "Tsukuba" formulation [61]:

$$
\begin{aligned}
D_{x, y}= & \delta_{x y}-\kappa_{f}\left[\left(1-\gamma_{t}\right) U_{x, t} \delta_{x+\hat{t}, y}+\left(1+\gamma_{t}\right) U_{x, t} \delta_{x+\hat{t}, y}\right] \\
& -\kappa_{f} \sum_{i}\left[\left(r_{s}-\nu_{s} \gamma_{i}\right) U_{x, t} \delta_{x+\hat{i}, y}+\left(r_{s}+\nu_{s} \gamma_{i}\right) U_{x, t} \delta_{x+\hat{i}, y}\right] \\
& -\kappa_{f}\left[c_{E} \sum_{i} F_{i t}(x) \sigma_{i t}+c_{B} \sum_{i, j} F_{i j}(x) \sigma_{i j}\right]
\end{aligned}
$$

The common approach of RHQ actions is to re-interpret the discretization effects and to retune the fully relativistic lattice action by introducing anisotropy in the valence sector to reproduce the correct dispersion relation, i.e., the equivalence between rest and kinetic mass, and physical spectrum. For our RHQ action, the tuning parameters have previously been computed for the ensembles studied here. The values of the parameters $\kappa_{f}, r_{s}, \nu_{s}, c_{E}$, and $c_{B}$ can be found in [64]. Meson masses using quark propagators computed from our implementation of this action are seen to be within $\sim 1 \%$ of the experimentally observed values, with splittings between, for example, $D$ and $D^{*}$ mesons equally well behaved.

For bottom quarks, as noted above, we employ the NRQCD action, as detailed in Appendix A. Overall, the NRQCD action is known to capture the relevant heavylight quark physics and account for relativistic effects at the few percent level [65-67]. 
TABLE V. Unphysical heavy quark masses used in our variablemass heavy anti-diquark investigation. Values are given divided by the physical bottom quark mass. For the definition of the entries in the two columns see Appendix A. The calculations were performed using the ensemble $E_{M}$ with one source position.

\begin{tabular}{lcc}
\hline \hline$a m_{Q}^{\prime}$ & $m_{Q}^{\prime} / m_{Q}$ & $m^{b^{\prime}} / m^{b}$ \\
\hline 0.9 & 0.466 & $0.594(3)$ \\
1.0 & 0.518 & $0.636(2)$ \\
1.2 & 0.622 & $0.680(5)$ \\
1.6 & 0.829 & $0.846(7)$ \\
3.0 & 1.554 & $1.463(12)$ \\
4.0 & 2.073 & $1.928(17)$ \\
8.0 & 4.145 & $4.395(35)$ \\
10.0 & 5.181 & $6.287(48)$ \\
\hline \hline
\end{tabular}

A list of the bare quark masses used in this study and the ratios of kinetic masses compared to the physical bottom quark is provided in Table V.

\section{INDICATIONS OF A BOUND $u d \bar{c} \bar{b}$ TETRAQUARK IN NATURE}

The successful phenomenological description of the heavy-quark mass dependence of $J^{P}=1^{+}$tetraquark binding energies described earlier identifies the $u d \bar{c} \bar{b}$ channel as the only such channel containing at least one charm antiquark likely to support a strong-interaction-stable bound tetraquark state. Given this insight, in this section, we focus our attention and resources on this channel and present results for the direct calculation of the mass of an $I\left(J^{P}\right)=0\left(1^{+}\right) u d \bar{c} \bar{b}$ tetraquark.

Our lattice results for the ground (red) and first excited (blue) state binding energies, obtained from the $3 \times 3$ GEVPs, are shown in Fig. 2. Results obtained using the corresponding $2 \times 2$ GEVPs, in which the local operator with $\bar{D}^{*} B$ discrete structure has been omitted, are shown for comparison in green, offset slightly in $t$ for presentational clarity.

Results shown in the left-hand panels are those obtained using the binding correlators Eq. (12). The results for $E_{H}$ show no evidence of the existence of a bound tetraquark state. For the $E_{M}$ and $E_{L}$ ensembles, however, one observes a clear rising behavior in both the $3 \times 3$ and $2 \times 2$ GEVP results, indicating the presence of a bound $u d \bar{c} \bar{b}$ tetraquark ground state for these ensembles. ${ }^{2}$

The right-hand panels of Fig. 2 focus on the binding energies themselves, which are shown in log-effective

\footnotetext{
${ }^{2}$ The apparent approximately linear rising behavior of the eigenvalues for $E_{M}$ and $E_{L}$ is a consequence of the weakness of the binding. For $t_{0}=4$, the central values of the ground state $E_{M}$ and $E_{L}$ binding energies (quoted below) correspond to maximum values 0.16 and 0.37 , respectively, of the argument, $|\Delta E|\left(t-t_{0}\right)$, of the exponential in Eq. (16) over the range of $t$ shown in the figure.
}

form, $\Delta E_{\text {eff }}(t)$, and given in physical units. The choice of log-effective form is for presentational purposes only; the binding energies shown by the shaded bands in the right-hand panels are those obtained through fits to the eigenvalues in the left-hand panels. The log-effective tetraquark energies to which these binding energies correspond have also been obtained directly from fits to the tetraquark correlation functions rather than to the binding correlators. The results of these fits are presented in Fig. 5 in Appendix C.

The data allow the first two binding correlator eigenvalues to be clearly resolved for each ensemble, with a signal that can be followed to $t / a \sim 16$. The overlaps with the chosen multiquark interpolating operators therefore seem good. The log-effective energies of the states corresponding to these eigenvalues, shown in the right panels of Fig. 5 in Appendix C, confirm that the signal is strong enough to reach the plateau regions of $a E_{\mathrm{eff}}(t)$. A comparison of the binding and tetraquark correlator results shows that the possibility anticipated above is, in fact, realized: the plateaus of the binding correlator are, in general, reached later than those of the tetraquark correlators. The binding correlator plateaus are therefore also shorter since the point of signal deterioration is the same for both the binding and tetraquark correlators. This is a sign of unwanted contamination from excited states in the meson denominator of the binding correlator ratio, as discussed in Sec. III. The longer plateaus for $a E_{\text {eff }}(t)$ in Fig. 5, however, confirm that, in spite of the shorter plateaus in the righthand panels of Fig. 2, the ground state plateau regions have been reached.

In all cases the third eigenvalue of the $3 \times 3$ GEVP (not shown here) is found to lie significantly higher than the first two, a fact confirmed by the eigenvalue plots in the lefthand panels of Fig. 5 in Appendix C.

Note that the results for the ground state eigenvalues obtained from the smaller $2 \times 2$ GEVP defined in Eq. (13), shown by the green vertical dashes in Fig. 2, agree very well with those obtained from the corresponding $3 \times 3$ GEVP. In contrast, the $2 \times 2$ results for the second eigenvalues, denoted by the green diagonal crosses, lie lower than those of the $3 \times 3$ analysis, and hence correspond to higher energies. This is as expected for analyses employing interpolating fields having a good overlap with the ground state, where both the $2 \times 2$ and $3 \times 3$ analyses should provide good access to the ground state energy, but the $2 \times 2$ analysis, where the second eigenvalue will have to represent not just the effects of the actual first excited state but also those of all higher excited states should yield results corresponding to higher energies than those obtained from the $3 \times 3$ analysis.

For the heavy pion ensemble, $E_{H}$, with $m_{\pi}=415 \mathrm{MeV}$, the ground state plateaus at the $\bar{D} B^{*}$ threshold, whereas already for $E_{M}$, with $m_{\pi}=299 \mathrm{MeV}$, the second eigenvalue is consistent with the threshold and the first 

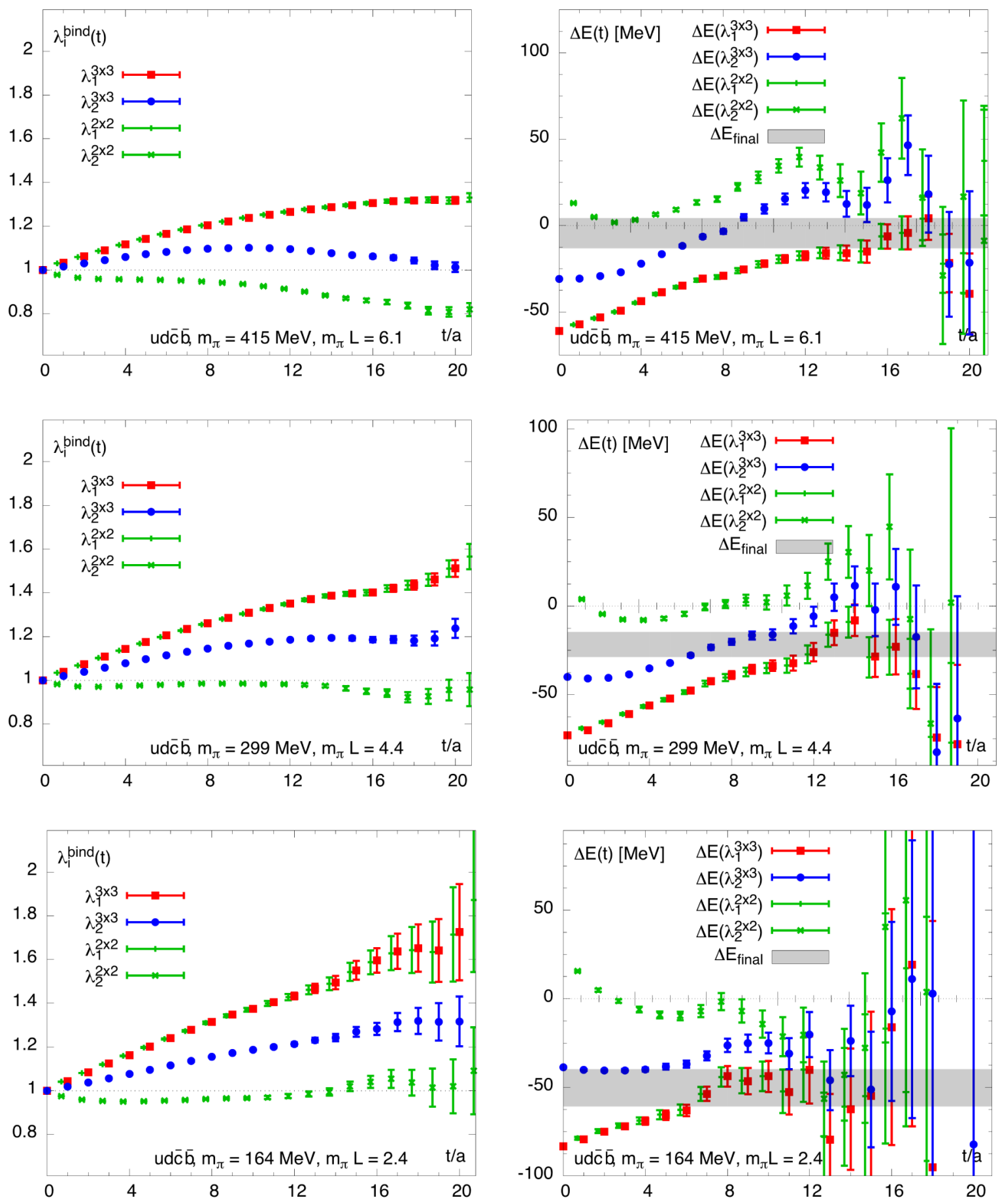

FIG. 2. $u d \bar{c} \bar{b}$ tetraquark results for binding correlator eigenvalues (left panels) and binding energies (right panels) on $E_{H}$ (top), $E_{M}$ (center), and $E_{L}$ (bottom). Red squares and blue circles denote $3 \times 3$ GEVP ground and first excited state results, respectively, green vertical dashes and green diagonal crosses ground and first excited state $2 \times 2$ GEVP results. The grey bands depict the final binding energies, derived from single-exponential fits to the first eigenvalues. Further details may be found in the text, and supplementary material in Appendix C. The $2 \times 2$ GEVP results are offset slightly in $t$ for visual clarity.

eigenvalue with a bound tetraquark interpretation. Finally, for $E_{L}$, with $m_{\pi}=164 \mathrm{MeV}$, both the first and second eigenvalues have central values corresponding to states below threshold. In light of the error bars, we suspect that, in this case, the second eigenvalue corresponds to a scattering state affected by the finite lattice volume rather than a genuine bound state. A more detailed investigation of this question must, however, await a future study involving sufficiently high-statistics ensembles with larger volumes for near-physical $m_{\pi}$. 
TABLE VI. Final estimated $u d \bar{c} \bar{b}$ binding energies. A negative value signals binding with respect to the $\bar{D} B^{*}$ threshold.

\begin{tabular}{lccc}
\hline \hline Ensemble & $m_{\pi}[\mathrm{MeV}]$ & $m_{\pi} L$ & $\Delta E_{u d \bar{c} \bar{b}}[\mathrm{MeV}]$ \\
\hline$E_{H}$ & 415 & 6.1 & $-4(9)$ \\
$E_{M}$ & 299 & 4.4 & $-22(7)$ \\
$E_{L}$ & 164 & 2.4 & $-50(11)$ \\
\hline \hline
\end{tabular}

To estimate the binding energies we have performed (uncorrelated) single-exponential fits Eq. (16) to the individual eigenvalues and accepted results satisfying $\chi^{2} /$ d.o.f. $\lesssim 1$. We emphasize that we perform fits to the eigenvalues of both the tetraquark correlators and binding correlators. In the former case, we subtract the sum of the threshold two-meson state masses to obtain the binding energy. All accepted results are compared and a result that is representative of both procedures is chosen. The final fit ranges are $t / a \in[10: 23]$ for $E_{L}, t / a \in[14: 20]$ for $E_{M}$, and $t / a \in[17: 21]$ for $E_{H}$. Further details of this procedure, as well as the resulting fit stabilities, are given in Appendix C. The final results obtained in this manner are listed in Table VI and plotted as the grey shaded bands in the righthand panels of Fig. 2.

Although further control of the dominant systematic uncertainties is necessary before an accurate prediction can be made, the results of our direct simulation provide good evidence for the existence of a strong-interaction-stable $J^{P}=1^{+} u d \bar{c} \bar{b}$ tetraquark state at physical $m_{\pi}$. We take the upper bound of the $E_{M}$ result and the lower bound of the $E_{L}$ result as providing the best assessment of the likely range of binding, leading to the expectation

$$
-61 \mathrm{MeV}<\Delta E_{u d \bar{c} \bar{b}}<-15 \mathrm{MeV}
$$

for the binding energy of the $u d \bar{c} \bar{b}$ tetraquark ground state relative to the $\bar{D} B^{*}$ threshold. For presentational simplicity in what follows, we will also quote this result in the equivalent compact form $\Delta E_{u d \bar{c} \bar{b}}=-38(23) \mathrm{MeV}$, using the midpoint of the range in Eq. (18) as the central value and half the width as the error estimate. This strategy for estimating the binding energy implied by our results appears plausible, given the ensembles currently available to us, since we expect both some deepening of the binding in going from $E_{M}$ to $E_{L}$ (as a consequence of the increasing spin-dependent, good-light-diquark attraction with decreasing light quark mass) and also potentially non-negligible finite-volume effects due to the small $m_{\pi} L$ of the $E_{L}$ ensemble. It thus seems reasonable to expect the true, infinite volume binding for physical $m_{\pi}$ to lie somewhere between the two bounds noted above. Our finite-volume systematic uncertainty, at present, clearly dwarfs the statistical errors on the individual measurements and only a careful finite-volume study will allow for a more accurate prediction. We are currently in the process of generating ensembles with larger volumes at near-physical $m_{\pi}$, and, once this is completed, we will report on the results obtained using these ensembles to more fully quantify the impact of finite-volume effects on the estimated physical- $m_{\pi}$ binding in a future work.

Taking PDG [68] values for the physical $D$ and $B^{*}$ masses, ${ }^{3}$ the binding energy estimate obtained above corresponds to a tetraquark mass of $\approx 7154(23) \mathrm{MeV}$.

For completeness, we also quote here the energy differences between the first excited state (corresponding to the second eigenvalue) and the $\bar{D} B^{*}$ threshold, which are 18(6) $\mathrm{MeV}$ for $E_{H}, 8(8) \mathrm{MeV}$ for $E_{M}$, and -26(7) MeV for $E_{L}$. These are compatible with our interpretation of the second eigenvalue as corresponding to the $\bar{D} B^{*}$ threshold for the $E_{M}$ and $E_{L}$ ensembles, with potentially nonnegligible finite-volume effects present in the $E_{L}$ case. One should, however, also bear in mind that the limited size of our basis of operators may impact how reliably we can extract the energy of the first excited state, particularly if this state corresponds to an infinite-volume scattering state and, as is likely, finite-volume effects are not negligible for the $E_{L}$ ensemble. A larger basis of operators and finitevolume analysis are thus desirable for a more robust study of the nature of this state.

\section{A. Discussion of systematic uncertainties}

In this calculation, the systematic error originating from the uncertainty of the lattice spacing in Table IV is negligible in comparison to the statistical error of the final result.

As noted above, we believe the dominant source of uncertainty in our result comes from finite-volume effects. The light-quark-mass dependence of the $u d \bar{c} \bar{b}$ masses covers multiple $m_{\pi} L$ and appears to support the interpretation of the ground states for the $E_{M}$ and $E_{L}$ ensembles as corresponding to genuine bound tetraquark states and the expectation that such a bound tetraquark state will therefore also exist at physical $m_{\pi}$. However, without additional ensembles with larger lattice volumes, a direct study of the scaling of the binding with $m_{\pi} L$, and a full extrapolation to infinite volume and physical $m_{\pi}$, is not feasible at present and must be left for future study.

Aside from general terms $\sim \exp \left(-m_{\pi} L\right)$, the finite lattice volume may affect particle energies in two ways. First, a bound state receives corrections proportional to $\exp (-|p| L)$, where $|p|$ is the binding momentum, defined via the energymomentum relation $-\Delta E=\sqrt{E_{1}^{2}+p^{2}}+\sqrt{E_{2}^{2}+p^{2}}-$ $E_{1}-E_{2}$, where $E_{1}$ and $E_{2}$ are the energies of the threshold particles. Second, a state which becomes a scattering state in the infinite-volume limit, but which lies below threshold at

\footnotetext{
${ }^{3}$ As the ensembles we use have identical $u$ and $d$ quark masses, and hence exact isospin symmetry, we use the average of the $D^{+}$ and $D^{0}$ masses for $m_{D}$.
} 

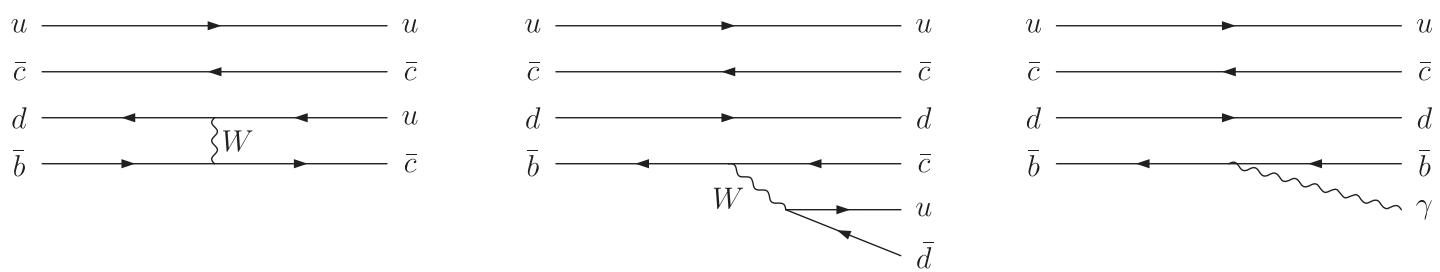

FIG. 3. Examples of potentially observable $u d \bar{c} \bar{b}$ tetraquark decay channels. Channels accessible if only weak decays are possible: (left) into two mesons (e.g., to $\bar{D}^{0} \bar{D}^{0}$ ), and (center) into three mesons (e.g., to $\pi^{+} \bar{D}^{0} D^{-}$). Right: decay to $\bar{D} B \gamma$, expected to dominate if electromagnetic decay is possible.

finite volume, may receive power-law corrections in $1 / L$, which for the $n=0$ states depend on $a_{0} / L$, where $a_{0}$ is the scattering length of the particles that define the two-meson threshold in the channel in question $[69,70]$.

Unlike the case of the $u d \bar{b} \bar{b}$ and $\ell s \bar{b} \bar{b}$ channels, where binding energies much larger than expected finite-volume effects were found [29], finite-volume effects may play a more important qualitative role in the $u d \bar{c} \bar{b}$ channel, where the expected binding and overall energy scale are lower. Even though a rigorous finite-volume study must be left for future work, we observe that, if the ground state energies for the $E_{M}$ and $E_{L}$ ensembles do, indeed, as the evidence above suggests, correspond to bound $u d \bar{c} \bar{b}$ tetraquark states, the binding momenta for these states would be $(|p|)_{L}=$ $373(171) \mathrm{MeV}$ and $(|p|)_{M}=245(140) \mathrm{MeV}$, respectively, implying a strong suppression of the finite-volume effects on the determined bound-state energies. Finite-volume studies with larger lattice volumes, however, remain desirable to more strongly test the bound-state interpretation of the ground states for the $E_{M}$ and $E_{L}$ ensembles.

\section{POSSIBLE EXPERIMENTAL DETECTION}

With a predicted binding of between 15 and $61 \mathrm{MeV}$ below the $\bar{D} B^{*}$ threshold, it is unclear whether the $J^{P}=$ $1^{+} u d \bar{c} \bar{b}$ tetraquark predicted by our results should, as its $u d \bar{b} \bar{b}$ and $\ell s \bar{b} \bar{b}$ analogues, be both strong- and electromagnetic-interaction stable, or be able to decay electromagnetically, to $\bar{D} B \gamma$, whose threshold lies $\sim 45 \mathrm{MeV}$ below $\bar{D} B^{*}$. In either case, such a state, with a mass $m_{u d c b} \simeq 7154(23) \mathrm{MeV}$, should be much more accessible to current experimental programs than are the corresponding doubly bottom states. ${ }^{4}$

Should the binding be greater than $\sim 45 \mathrm{MeV}$, the $u d \bar{c} \bar{b}$ tetraquark will decay only weakly and suffer from the experimental disadvantage of having a large number of exclusive decay modes, each with a relatively small branching fraction. Such decay modes would, however, have the compensating advantage of being accompanied by

\footnotetext{
${ }^{4}$ It is worth mentioning that, even for the more experimentally challenging doubly bottom tetraquarks, a recent study of possible branching fractions came to a positive conclusion regarding their discovery potential in current experiments [71].
}

secondary heavy-meson decays with displaced vertices. The left and center panels of Fig. 3 show examples of such decays. Two- or three-meson decays such as those to $\left(\bar{D}^{0} \bar{D}^{0}\right)$ or $\pi^{+} \bar{D}^{0} D^{-}$might then serve as useful potential experimental signatures. Another three-body mode potentially suitable for detection, produced if the $W^{+}$materializes as a $c \bar{s}$ rather than a $u \bar{d}$ pair, is $J / \psi \bar{D}^{0} K^{0}$.

In contrast, should the $u d \bar{c} \bar{b}$ binding energy be less than $\sim 45 \mathrm{MeV}$, the state should decay electromagnetically essentially $100 \%$ of the time to $\bar{D} B \gamma$, as in the rightmost panel of Fig. 3.

With (given the predicted binding energy) less than $\sim 20 \mathrm{MeV}$ of phase space available to the electromagnetic decay, such a tetraquark, produced with a reasonable momentum in the lab frame, will decay to produce a mixed-heavy-flavor $D^{+} B^{-}$or $\bar{D}^{0} B^{0}$ meson pair highly collimated in the lab frame.

\section{CONCLUSIONS}

The study of heavy tetraquarks of the $q q^{\prime} \bar{Q}^{\prime} \bar{Q}$ type continues to yield insight into the binding of exotic hadrons. This paper provides evidence of a stronginteraction-stable $I\left(J^{P}\right)=0\left(1^{+}\right) u d \bar{c} \bar{b}$ tetraquark which we estimate to lie $15-61 \mathrm{MeV}$ below the corresponding noninteracting, two-meson $\left(\bar{D} B^{*}\right)$ threshold. The predicted mass range, $\approx 7154(23) \mathrm{MeV}$, thus straddles the electromagnetic $\bar{D} B \gamma$ decay threshold, making it impossible, at present, to predict whether it will decay electromagnetically or only weakly. With $B_{c}$ production, and hence the simultaneous production of $b \bar{b}$ and $c \bar{c}$ pairs, already established experimentally, these results clearly motivate a search for this state at $\mathrm{LHCb}$.

The results of the direct computation above further strengthen the argument that this class of tetraquarks becomes more strongly bound as the light-quark component becomes lighter. The variable, unphysical heavy-quark mass study also confirms the expectation that such tetraquarks become less bound as the heavy anti-diquark reduced mass decreases.

Complementary to the direct computation of the binding of the $I\left(J^{P}\right)=0\left(1^{+}\right) u d \bar{c} \bar{b}$ tetraquark, the NRQCD study of the $u d \bar{b}^{\prime} \bar{b}^{\prime}, u d \bar{b}^{\prime} \bar{b}, \ell s \bar{b}^{\prime} \bar{b}^{\prime}$, and $\ell s \bar{b}^{\prime} \bar{b}$ channels with variable heavy quark mass, $m_{b^{\prime}}$, extending down to $0.60 m_{b}$ 
confirms our qualitative understanding of the basic physics responsible for the observed tetraquark binding. This study also suggests that strong-interaction-stable $u d \bar{c} \bar{c}, \ell s \bar{c} \bar{b}$, and $\ell s \bar{c} \bar{c}$ tetraquarks are unlikely to exist (see also [40]), though direct computations using a relativistic charm action at almost physical light quark masses have yet to be carried out for these channels.

Our prediction for the $u d \bar{c} \bar{b}$ binding has an error dominated by what we believe to be a conservative estimate of the finite-volume systematic. While statistics does not appear to be a problem, having more operators and/or crafting sources and sinks that better overlap with our desired ground states should provide longer plateaus. In the future, futher investigations of the $u d \bar{c} \bar{b}$ channel using ensembles with additional near-physical light pion masses and larger spatial volumes will allow us to obtain better quantitative control of the extrapolation to physical $m_{\pi}$ and finite-volume effects.

\section{ACKNOWLEDGMENTS}

Propagator inversions for this work were performed on the compute cluster "GPC" at SciNet, Toronto. This work was supported in part by the Natural Sciences and Engineering Research Council (NSERC) of Canada. The calculations were performed as part of an RAC allocation under the Compute Canada initiative.

\section{APPENDIX A: OUR IMPLEMENTATION OF THE NRQCD FRAMEWORK}

We use the NRQCD Hamiltonian [72-74]

$$
\begin{aligned}
H_{0}= & -c_{0} \frac{\Delta^{(2)}}{2 m_{Q}}, \\
\delta H= & -c_{1} \frac{\left(\Delta^{(2)}\right)^{2}}{8 m_{Q}^{3}}+\frac{c_{2}}{U_{0}^{4}} \frac{i g}{8 m_{Q}^{2}}(\tilde{\boldsymbol{\Delta}} \cdot \tilde{\boldsymbol{E}}-\tilde{\boldsymbol{E}} \cdot \tilde{\boldsymbol{\Delta}}) \\
& -\frac{c_{3}}{U_{0}^{4}} \frac{g}{8 m_{Q}^{2}} \boldsymbol{\sigma} \cdot(\tilde{\boldsymbol{\Delta}} \times \tilde{\boldsymbol{E}}-\tilde{\boldsymbol{E}} \times \tilde{\boldsymbol{\Delta}}) \\
& -\frac{c_{4}}{U_{0}^{4}} \frac{g}{2 m_{Q}} \boldsymbol{\sigma} \cdot \tilde{\boldsymbol{B}}+c_{5} \frac{a^{2} \Delta^{(4)}}{24 m_{Q}}-c_{6} \frac{a\left(\Delta^{(2)}\right)^{2}}{16 n m_{Q}^{2}},
\end{aligned}
$$

where $\tilde{\boldsymbol{E}}, \tilde{\boldsymbol{B}}$, and $\tilde{\boldsymbol{\Delta}}$ denote the $O(a)$-improved color electric field, color magnetic field, and spatial lattice derivative, respectively. $\Delta^{(2)}$ is the lattice Laplacian, $g$ is the bare gauge coupling, and $n$ is a mode number used in the evolution equation [see Eq. (A4) below]. We find $n=4$ to be a reasonable value for this stability parameter.

Here, the tadpole-improvement coefficient $U_{0}$ is set via the fourth root of the plaquette, and the coefficients $c_{i}$ are assigned their tree-level values of 1 . This leaves one free parameter, $m_{Q}$, to be tuned to give the desired heavy-quark physics. This tuning is achieved by measuring the slope of the dispersion relation, and hence the "kinetic" mass, of the spin-averaged $\Upsilon$ and $\eta_{b}$ on Fourier-transformed local-local current correlators. Explicitly, with

$$
\begin{aligned}
\eta_{b}\left(M_{0}, p^{2}\right) & =\eta_{b}\left(M_{0}\right)+\frac{p^{2}}{2 m_{\eta}^{b}}, \\
\Upsilon\left(M_{0}, p^{2}\right) & =\Upsilon\left(M_{0}\right)+\frac{p^{2}}{2 m_{\Upsilon}^{b}},
\end{aligned}
$$

we take

$$
m^{b}=\frac{1}{4}\left(m_{\eta}^{b}+3 m_{\Upsilon}^{b}\right) .
$$

With the parameters of the NRQCD action all fixed, the evolution equation,

$$
\begin{aligned}
G(x, t+1)= & \left(1-\frac{H_{0}}{2 n}\right)^{n}\left(1-\frac{\delta H}{2}\right) \frac{U_{t}^{\dagger}(x)}{U_{0}}\left(1-\frac{\delta H}{2}\right) \\
& \times\left(1-\frac{H_{0}}{2 n}\right)^{n} G(x, t),
\end{aligned}
$$

where $U_{t}$ denotes a gauge link in the temporal direction, is used to compute our heavy quark propagators. Static heavy quark propagators can be realized by setting all the coefficients $c_{i}$ in this action to 0 .

The NRQCD action can be organized as an expansion in $1 / m_{Q}$, and naively its regime of validity is limited to the region $m_{Q} \gtrsim 1$ in lattice units.

\section{APPENDIX B: DETAILS OF THE VARIABLE-HEAVY-MASS STUDY}

This study focuses on unphysical bottom quarks using the ensemble $E_{M}$ of Table IV with a single source position. Visible effects due to violations of the condition $a m_{Q} \gtrsim 1$ are e.g., the increase of the hyperfine splitting. While tunings of the parameters $c_{i}$ beyond tree level, as well as the inclusion of additional terms in the action, and/or an increase in the stability parameter $n$, could be considered to expand the region of validity of the NRQCD approximation, here we choose to work with tree-level values and the form of the NRQCD Hamiltonian detailed in Appendix A. Monitoring the hyperfine splitting, we find that $m_{Q} \simeq 0.594 m_{b}$ appears to be the lowest acceptable value for our calculation. From extrapolation of our heavyquark data, the charm quark lies at around $m_{c} \simeq 0.33 m_{b}$ and is thus beyond our reach in the NRQCD approach.

The binding energies of the $u d \bar{b}^{\prime} \bar{b}^{\prime}, u d \bar{b}^{\prime} \bar{b}, \ell s \bar{b}^{\prime} \bar{b}^{\prime}$, and $\ell s \bar{b}^{\prime} \bar{b}$ tetraquark states on the $E_{M}$ ensemble are extracted by fitting the smallest eigenvalue of the respective GEVPs to a single-exponential ansatz. All fit results with $\chi^{2} /$ d.o.f. $\leq 1$ are kept for further analysis. The fit procedure does not take into account correlations in the data. The final numbers are chosen as representative results with the longest fit window possible.

The resulting data and fits are shown in the left panels of Fig. 4. We observe positive exponential growth of the first eigenvalues determined from the binding correlator with Euclidean time $t$. This growth becomes steeper as the heavy 

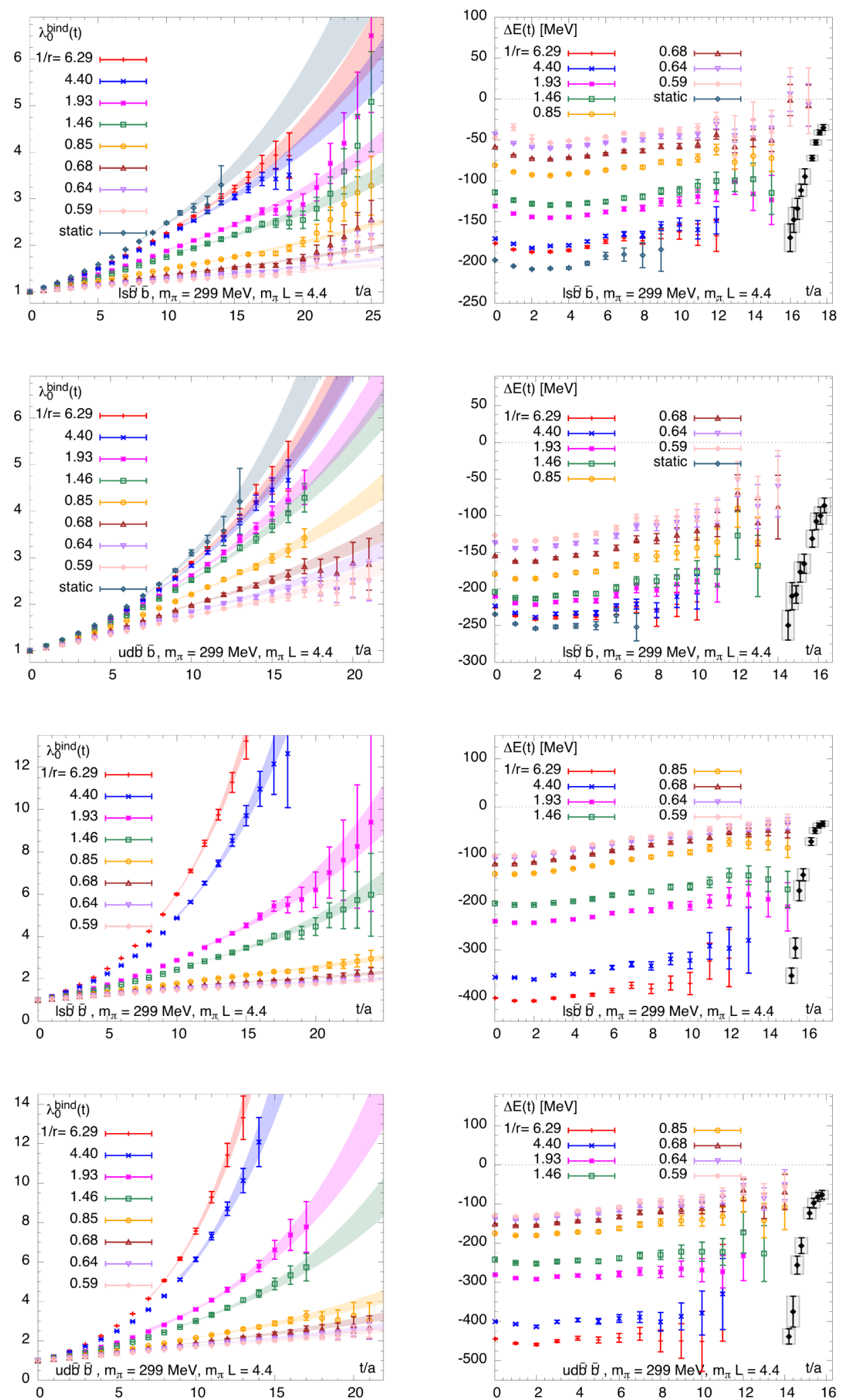

FIG. 4. Binding correlators (left) and effective energies (right) for the four channels $\ell s \bar{b}^{\prime} \bar{b}, u d \bar{b}^{\prime} \bar{b}, \ell s \bar{b}^{\prime} \bar{b}^{\prime}$, and $u d \bar{b}^{\prime} \bar{b}^{\prime}$ (top to bottom). Fit results are given as shaded bands (left) or boxes (right). 
quark mass is increased in all four tetraquark channels (especially so for the equal-heavy-mass case, where the binding energy is unbounded as $m_{b^{\prime}} \rightarrow \infty$ ). The shaded bands denote the corresponding fits and are seen to describe the data well. We also determine the effective binding energies and show these results (data), together with the corresponding binding energy fits (shaded boxes) in the right panels of Fig. 4. The success of the phenomenological fit forms detailed in Sec. II in reproducing these lattice data confirms our understanding of the basic binding mechanism.

\section{APPENDIX C: FURTHER DETAILS OF THE $u d \bar{c} \bar{b}$ ANALYSIS}

In addition to the direct determination of our tetraquark binding energies from fits to binding correlator data, we have also estimated the binding energies via (uncorrelated)
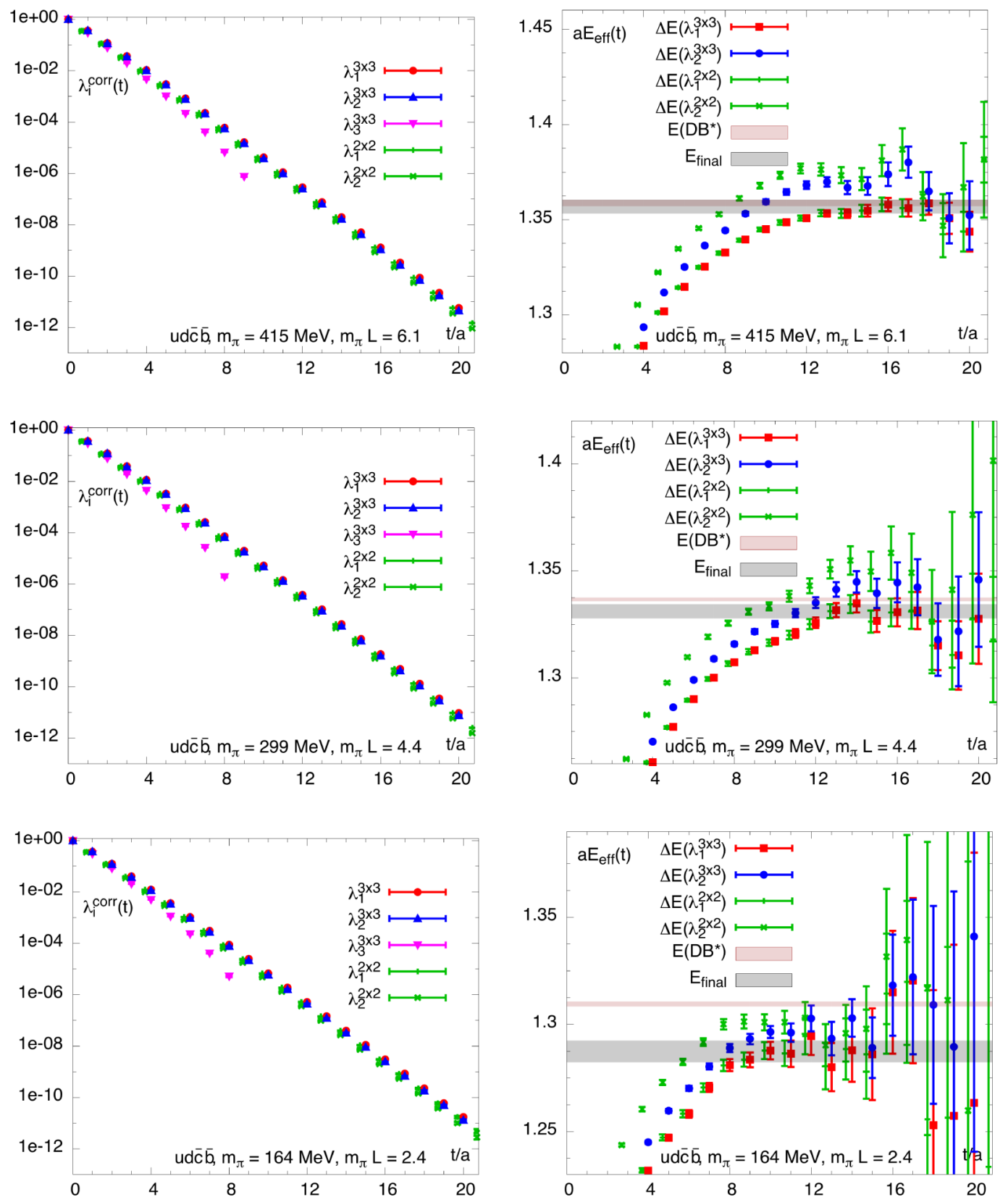

FIG. 5. $u d \bar{c} \bar{b}$ tetraquark results on $E_{H}$ (top), $E_{M}$ (center), and $E_{L}$ (bottom). The left panels show the GEVP eigenvalues, and the right panels the corresponding energies. The red and blue symbols show, respectively, the ground and first excited state results obtained from the $3 \times 3$ GEVP analyses. The green vertical dashes and green diagonal crosses similarly denote the ground and first excited state results obtained from the $2 \times 2$ GEVP analyses. The two-meson $\bar{D} B^{*}$ thresholds, shown for comparison, are given by the brown bands in the right-hand panels. The grey bands in those same panels depict the final energies obtained from the single exponential fits to the eigenvalues described above. Further details are given in the text. The $2 \times 2$ GEVP results have been offset in $t$ for visual clarity. 
single-exponential fits of the ground-state tetraquark energies to the eigenvalue data for the tetraquark correlation functions. The tetraquark eigenvalue data are shown in the left-hand panels of Fig. 5 and the corresponding
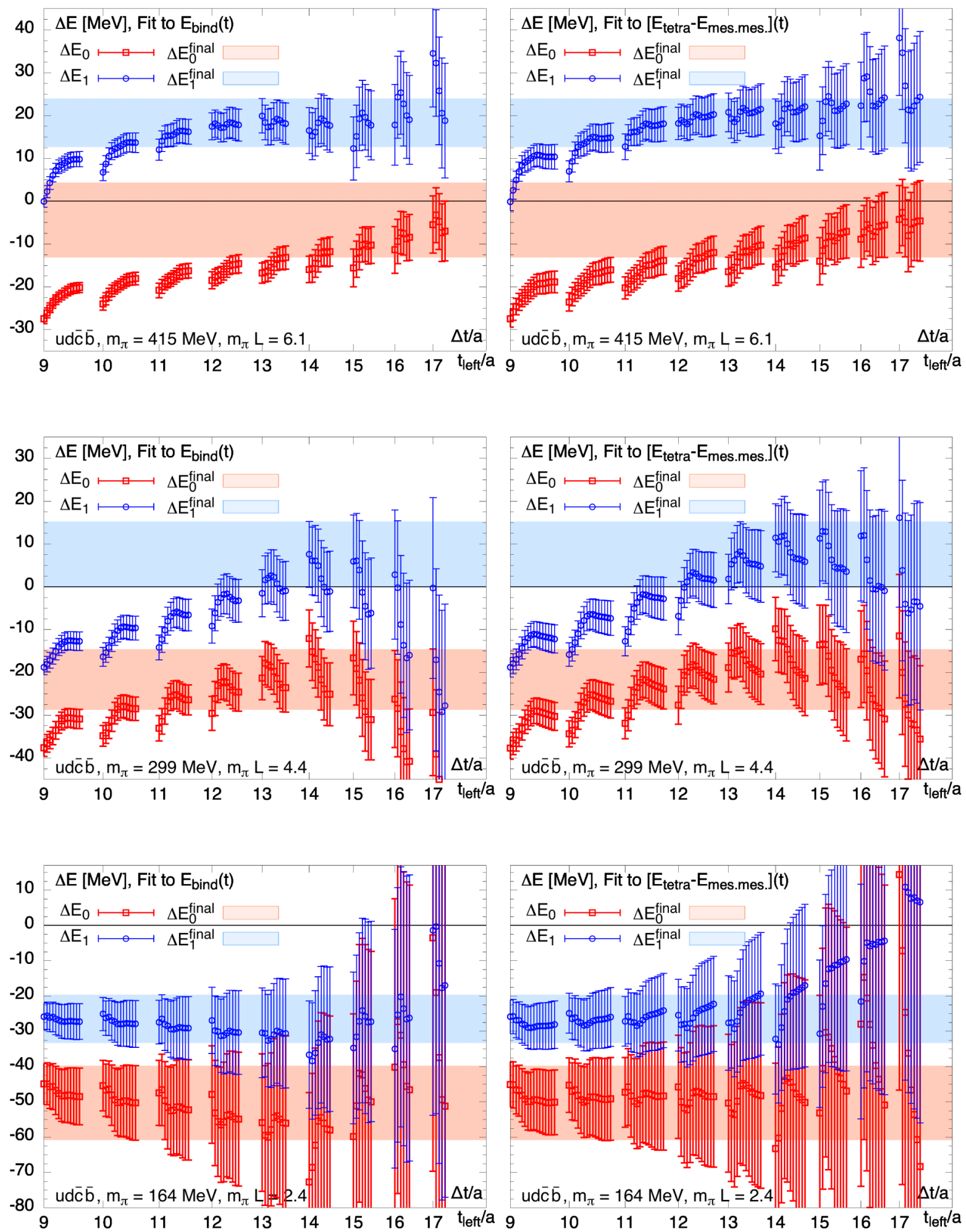

FIG. 6. Fit window dependence for fitting the binding correlator GEVP solutions and those obtained from the particle correlators for the ensembles $E_{H}$ (top), $E_{M}$ (center), and $E_{L}$ (bottom). We obtain our final results from this selection of accepted fits in the stable region that is representative of both procedures. 
GEVP are specified using green vertical dashes and green diagonal crosses.

The same fit strategy is employed to extract the binding energies from the binding correlator data and the tetraquark energies themselves from the tetraquark correlator data. Explicitly, we select fit windows in Euclidean $t$ extending from $t_{\text {left }}$ to $t_{\text {right }}=t_{\text {left }}+\Delta t$ whose results satisfy $\chi^{2} /$ d.o.f. $\lesssim 1$. In the case of the binding correlator, the fit immediately gives the binding energy. In the case of the four-quark "diquark-diquark" and "meson-meson" correlators, the two meson threshold mass sum is subtracted from the resulting tetraquark mass. The accepted results obtained in this way are shown as a function of $t_{\text {left }}$ in the right panels of Fig. 6, and the corresponding results obtained using the binding correlator GEVPs in the corresponding left panels. The multiple points plotted for each $t_{\text {left }}$ are those corresponding to different choices of $\Delta t$. From this selection of results the final, quoted numbers (corresponding to the grey bands in Fig. 5) are chosen in such a way that they lie in the stable regions of the plots, and are representative of both procedures. The final fit ranges used are $t / a \in[10: 23]$ for $E_{L}, t / a \in[14: 20]$ for $E_{M}$, obtained from the left panels, and $t / a \in[17: 21]$ for $E_{H}$ chosen from the right panels.
[1] A. Ali, J. S. Lange, and S. Stone, Prog. Part. Nucl. Phys. 97, 123 (2017).

[2] R. Aaij et al. (LHCb Collaboration), Phys. Rev. Lett. 115, 072001 (2015).

[3] S. Zouzou, B. Silvestre-Brac, C. Gignoux, and J. M. Richard, Z. Phys. C 30, 457 (1986).

[4] H. J. Lipkin, Phys. Lett. B 172, 242 (1986).

[5] B. Silvestre-Brac and C. Semay, Z. Phys. C 57, 273 (1993).

[6] C. Semay and B. Silvestre-Brac, Z. Phys. C 61, 271 (1994).

[7] S. Pepin, F. Stancu, M. Genovese, and J. M. Richard, Phys. Lett. B 393, 119 (1997).

[8] D. M. Brink and F. Stancu, Phys. Rev. D 57, 6778 (1998).

[9] T. Barnes, N. Black, D. J. Dean, and E. S. Swanson, Phys. Rev. C 60, 045202 (1999).

[10] B. A. Gelman and S. Nussinov, Phys. Lett. B 551, 296 (2003).

[11] J. Vijande, F. Fernandez, A. Valcarce, and B. Silvestre-Brac, Eur. Phys. J. A 19, 383 (2004).

[12] D. Janc and M. Rosina, Few Body Syst. 35, 175 (2004).

[13] D. Ebert, R. N. Faustov, V. O. Galkin, and W. Lucha, Phys. Rev. D 76, 114015 (2007).

[14] J. Vijande, E. Weissman, A. Valcarce, and N. Barnea, Phys. Rev. D 76, 094027 (2007).

[15] M. Zhang, H. X. Zhang, and Z. Y. Zhang, Commun. Theor. Phys. 50, 437 (2008).

[16] S. H. Lee and S. Yasui, Eur. Phys. J. C 64, 283 (2009).

[17] J. Vijande, A. Valcarce, and N. Barnea, Phys. Rev. D 79, 074010 (2009).

[18] Y. Yang, C. Deng, J. Ping, and T. Goldman, Phys. Rev. D 80, 114023 (2009).

[19] A. Valcarce, J. Vijande, and T. F. Carames, Int. J. Mod. Phys. Conf. Ser. 02, 173 (2011).

[20] T. F. Carames, A. Valcarce, and J. Vijande, Phys. Lett. B 699, 291 (2011).

[21] S. Ohkoda, Y. Yamaguchi, S. Yasui, K. Sudoh, and A. Hosaka, Phys. Rev. D 86, 034019 (2012).

[22] T. Hyodo, Y.-R. Liu, M. Oka, K. Sudoh, and S. Yasui, Phys. Lett. B 721, 56 (2013).

[23] R. R. Silbar and T. Goldman, Int. J. Mod. Phys. E 23, 1450091 (2014).
[24] M. Karliner and J. L. Rosner, Phys. Rev. Lett. 119, 202001 (2017).

[25] E. J. Eichten and C. Quigg, Phys. Rev. Lett. 119, 202002 (2017).

[26] M.-L. Du, W. Chen, X.-L. Chen, and S.-L. Zhu, Phys. Rev. D 87, 014003 (2013).

[27] W. Chen, T. G. Steele, and S.-L. Zhu, Phys. Rev. D 89, 054037 (2014).

[28] P. Bicudo, K. Cichy, A. Peters, B. Wagenbach, and M. Wagner, Phys. Rev. D 92, 014507 (2015).

[29] A. Francis, R. J. Hudspith, R. Lewis, and K. Maltman, Phys. Rev. Lett. 118, 142001 (2017).

[30] R. L. Jaffe, Phys. Rep. 409, 1 (2005).

[31] D. G. Richards, D. K. Sinclair, and D. W. Sivers, Phys. Rev. D 42, 3191 (1990).

[32] A. Mihaly, H. R. Fiebig, H. Markum, and K. Rabitsch, Phys. Rev. D 55, 3077 (1997).

[33] C. Stewart and R. Koniuk, Phys. Rev. D 57, 5581 (1998).

[34] C. Michael and P. Pennanen (UKQCD Collaboration), Phys. Rev. D 60, 054012 (1999).

[35] P. Pennanen, C. Michael, and A. M. Green, Nucl. Phys. B, Proc. Suppl. 83, 200 (2000).

[36] M. S. Cook and H. R. Fiebig, arXiv:hep-lat/0210054.

[37] W. Detmold, K. Orginos, and M. J. Savage, Phys. Rev. D 76, 114503 (2007).

[38] G. Bali and M. Hetzenegger (QCDSF Collaboration), Proc. Sci., LATTICE2011 (2011) 123.

[39] M. Wagner (ETM Collaboration), Acta Phys. Pol. B Proc. Suppl. 4, 747 (2011).

[40] G. K. C. Cheung, C. E. Thomas, J. J. Dudek, and R. G. Edwards (Hadron Spectrum Collaboration), J. High Energy Phys. 11 (2017) 033.

[41] Z. S. Brown and K. Orginos, Phys. Rev. D 86, 114506 (2012).

[42] P. Bicudo and M. Wagner (ETM Collaboration), Phys. Rev. D 87, 114511 (2013).

[43] P. Bicudo, J. Scheunert, and M. Wagner, Proc. Sci., LATTICE2016 (2016) 103.

[44] R. Aaij et al. (LHCb Collaboration), Phys. Rev. Lett. 119, 112001 (2017). 
[45] A. Czarnecki, B. Leng, and M. B. Voloshin, Phys. Lett. B 778, 233 (2018).

[46] T. Mehen, Phys. Rev. D 96, 094028 (2017).

[47] L. Heller and J.A. Tjon, Phys. Rev. D 32, 755 (1985).

[48] J. Carlson, L. Heller, and J. A. Tjon, Phys. Rev. D 37, 744 (1988).

[49] A. V. Manohar and M. B. Wise, Nucl. Phys. B399, 17 (1993).

[50] T. Iritani, S. Aoki, T. Doi, T. Hatsuda, Y. Ikeda, T. Inoue, N. Ishii, H. Nemura, and K. Sasaki, Phys. Rev. D 96, 034521 (2017).

[51] A. Francis, R. J. Hudspith, R. Lewis, and K. Maltman, Proc. Sci., LATTICE2016 (2016) 132.

[52] Y. Namekawa et al. (PACS-CS Collaboration), Phys. Rev. D 87, 094512 (2013).

[53] R. J. Hudspith, A. Francis, R. Lewis, and K. Maltman, Proc. Sci., LATTICE2016 (2017) 133.

[54] S. Aoki et al. (PACS-CS Collaboration), Phys. Rev. D 79, 034503 (2009).

[55] B. Sheikholeslami and R. Wohlert, Nucl. Phys. B259, 572 (1985).

[56] Y. Iwasaki, Nucl. Phys. B258, 141 (1985).

[57] C. B. Lang, L. Leskovec, D. Mohler, S. Prelovsek, and R. M. Woloshyn, Phys. Rev. D 90, 034510 (2014).

[58] R. J. Hudspith (RBC and UKQCD Collaborations), Comput. Phys. Commun. 187, 115 (2015).

[59] M. Lüscher, Comput. Phys. Commun. 165, 199 (2005).
[60] A. X. El-Khadra, A. S. Kronfeld, and P. B. Mackenzie, Phys. Rev. D 55, 3933 (1997).

[61] S. Aoki, Y. Kuramashi, and S.-I. Tominaga, Prog. Theor. Phys. 109, 383 (2003).

[62] N. H. Christ, M. Li, and H.-W. Lin, Phys. Rev. D 76, 074505 (2007).

[63] M. B. Oktay and A. S. Kronfeld, Phys. Rev. D 78, 014504 (2008).

[64] Y. Namekawa et al. (PACS-CS Collaboration), Phys. Rev. D 84, 074505 (2011).

[65] R. Lewis and R. M. Woloshyn, Phys. Rev. D 79, 014502 (2009).

[66] A. Gray, I. Allison, C. T. H. Davies, E. Dalgic, G. P. Lepage, J. Shigemitsu, and M. Wingate, Phys. Rev. D 72, 094507 (2005).

[67] Z. S. Brown, W. Detmold, S. Meinel, and K. Orginos, Phys. Rev. D 90, 094507 (2014).

[68] C. Patrignani et al. (Particle Data Group), Chin. Phys. C 40, 100001 (2016).

[69] M. Luscher, Commun. Math. Phys. 105, 153 (1986).

[70] M. Luscher, Nucl. Phys. B354, 531 (1991).

[71] A. Ali, Q. Qin, and W. Wang, Phys. Lett. B 785, 605 (2018).

[72] B. A. Thacker and G. P. Lepage, Phys. Rev. D 43, 196 (1991).

[73] G. P. Lepage, L. Magnea, C. Nakhleh, U. Magnea, and K. Hornbostel, Phys. Rev. D 46, 4052 (1992).

[74] C. T. H. Davies, K. Hornbostel, A. Langnau, G. P. Lepage, A. Lidsey, J. Shigemitsu, and J. H. Sloan, Phys. Rev. D 50, 6963 (1994). 